\title{
Influência de sintomas gastrointestinais na qualidade de vida em crianças portadoras do Transtorno do Espectro Autista
}

\author{
Influence of gastrointestinal symptoms on quality of life in children with Autism Spectrum \\ Disorder
}

\author{
Influencia de los síntomas gastrointestinales en la calidad de vida en niños con Trastorno \\ del Espectro Autista
}

Pedro Antonio Rodrigues Dias ${ }^{1 *}$, Elisa Ribeiro Martins ${ }^{2}$, Júlia Incau Guazzelli ${ }^{3}$, Kathlyn Cristina Canedo Póvoa ${ }^{4}$, Letícia Melo Berbary da Silva ${ }^{5}$, Luiza Bomtempo Araújo ${ }^{6}$, Maria Grasiele dos Anjos Gois $^{7}$, Marina Déda Peixoto Leite ${ }^{8}$, Vitor Michelli Leitão Rodrigues ${ }^{9}$, Maianna Viana Almeida Aguiar $^{10}$.

\section{RESUMO}

Objetivo: Avaliar como as alterações gastrointestinais afetam a qualidade de vida das crianças com Transtorno do Espectro Autista (TEA). Revisão bibliográfica: Crianças do espectro autista apresentam seletividade alimentar e se limitam a uma alimentação restrita a cheiros, cores, sabores e temperaturas, tendendo a forte preferência por carboidratos e alimentos processados, o que pode levar a um alto índice de obesidade nesse grupo. Com base em estudos já feitos, crianças com TEA apresentam quatro vezes mais sintomas gastrointestinais associados a um comprometimento da microbiota intestinal, quando comparadas a crianças com desenvolvimento típico. Com a desregulação do eixo microbiota-intestino-cérebro, pode ocorrer alterações cognitivos comportamentais, favorecendo sintomas como ansiedade, estresse e alterações do humor. Considerações finais: Visando diminuir a influência de desconfortos gastrointestinais na qualidade de vida do paciente, a terapia nutricional com alimentos frequentemente deficientes em crianças com TEA, pode ser uma opção de tratamento eficiente para sintomas gastrointestinais e comportamentais, ainda que essa terapia seja dificultada pela alta seletividade alimentar nessas crianças. Entretanto, mais estudos são necessários para compreender melhor quais dietas trariam melhores benefícios juntos a maior adesão ao tratamento.

Palavras-chave: Transtorno do Espectro Autista, Microbioma gastrointestinal, Qualidade de vida.

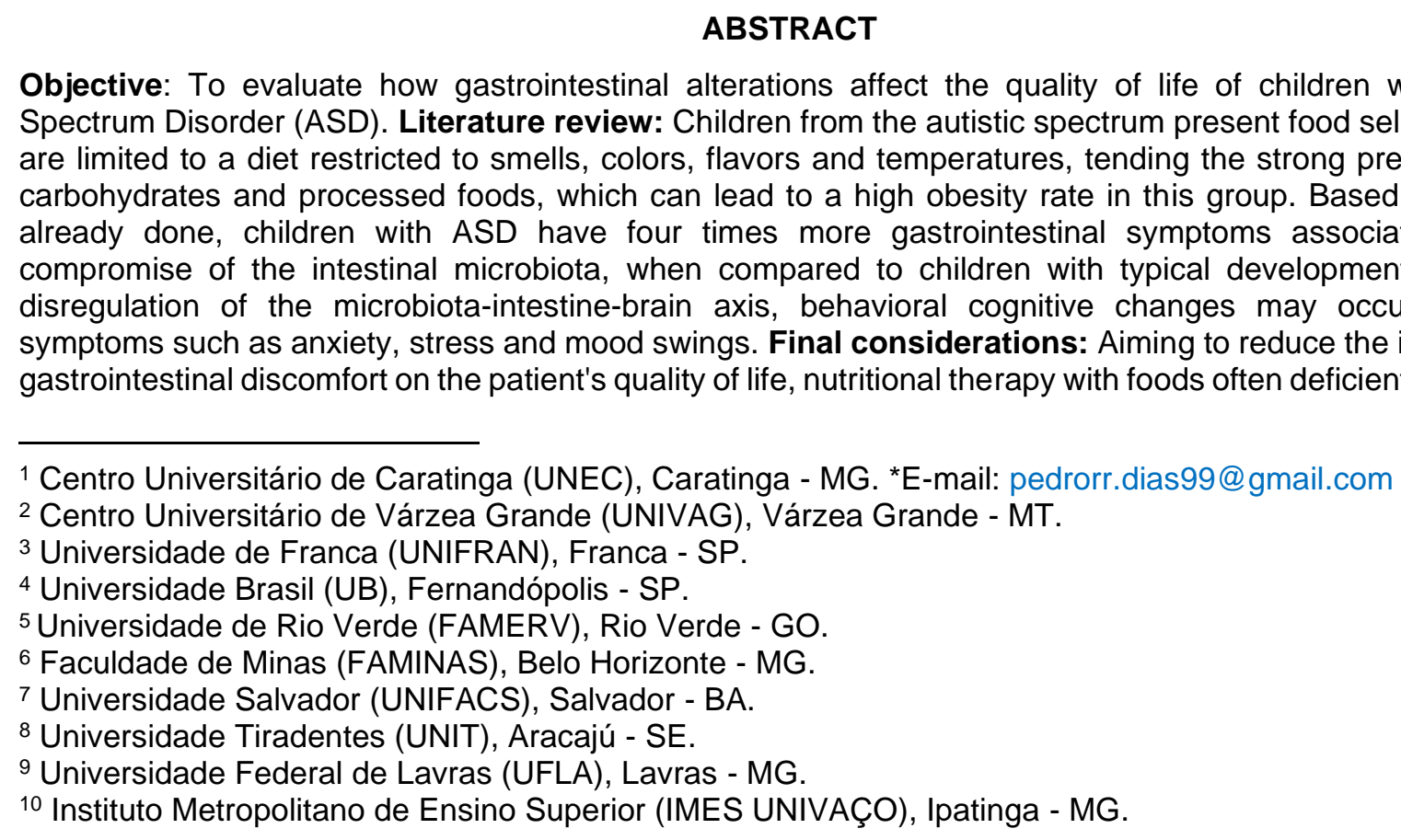


with ASD may be an efficient treatment option for gastrointestinal and behavioral symptoms, although this therapy is hampered by high food selectivity in these children. However, more studies are needed to better understand which diets would bring better benefits together to greater treatment adhering.

Key words: Autism Spectrum Disorder, Gastrointestinal microbiome, Quality of life.

\section{RESUMEN}

Objetivo: Evaluar cómo las alteraciones gastrointestinales afectan la calidad de vida de los niños con Trastorno del Espectro Autista (TEA). Revisión de la literatura: Los niños del espectro autista presentan selectividad alimentaria y se limitan a una dieta restringida a olores, colores, sabores y temperaturas, que tiende la fuerte preferencia por los carbohidratos y los alimentos procesados, lo que puede conducir a una alta tasa de obesidad en este grupo. Según los estudios ya realizados, los niños con TEA tienen cuatro veces más síntomas gastrointestinales asociados con un compromiso de la microbiota intestinal, en comparación con los niños con desarrollo típico. Con la desregulación del eje microbiota-intestino-cerebro, pueden ocurrir cambios cognitivos conductuales, favoreciendo síntomas como ansiedad, estrés y cambios de humor. Consideraciones finales: Con el objetivo de reducir la influencia de las molestias gastrointestinales en la calidad de vida del paciente, la terapia nutricional con alimentosa menudo deficiente en niños con TEA, puede ser una opción de tratamiento eficiente para los síntomas gastrointestinales y conductuales, aunque esta terapia se ve obstaculizada por la alta selectividad alimentaria en estos niños. Sin embargo, se necesitan más estudios para entender mejor qué dietas aportarían mejores beneficios para una mayor adherente del tratamiento.

Palabras clave: Trastorno del Espectro Autista, Microbioma gastrointestinal, Calidad de vida.

\section{INTRODUÇÃO}

Em todo o mundo, estima-se que 1 em cada 160 crianças apresenta algum grau de Transtorno do Espectro Autista (TEA), com prevalência quatro vezes maior em meninos do que em meninas. Com seu início na infância e tendência a persistir até a vida adulta, o TEA se refere a uma série de condições caracterizadas por algum grau de comprometimento na interação social, na comunicação, na linguagem e por padrões repetitivos de comportamento. Relativo a isso, há algumas décadas, acreditava-se que o TEA seria majoritariamente $(90 \%)$ relacionado a fatores genéticos e hereditários. Porém, estudos recentes descrevem uma herdabilidade genética de 50\% e uma forte relação com fatores ambientais (ROUSSIN L, et al., 2020).

Alguns portadores desse transtorno podem viver de forma independente, porém outros necessitam de cuidados e apoio ao longo da vida em decorrência de várias incapacidades, segundo a Organização Mundial da Saúde (OMS, 2017). Crianças com autismo tem seletividade alimentar e dietas limitadas devido ao cheiro, sabor, cor e temperatura dos alimentos.

Dessa forma, são determinadas rotinas alimentares e padrões de ingestão alimentar que influenciam a recusa de alimentos com consequente deficiência ou até mesmo excessos de alguns nutrientes. Observa-se que crianças possuem uma forte preferência por alimentos ricos em amidos, lanches e alimentos processados e rejeição de frutas, vegetais ou proteínas, podendo levar a um ganho de peso excessivo, com as consequentes altas taxas de obesidade em crianças com autismo (PERETTI S, et al., 2018).

Logo, uma ingestão nutricional adequada representa um desafio para crianças com TEA devido a comportamentos alimentares problemáticos, mas também em decorrência de sintomas gastrointestinais, suscetibilidade a alergias alimentares e anormalidades metabólicas. Além disso, sabe-se que a microbiota intestinal, hospedada pelo trato gastrointestinal, é definida como uma população complexa e específica de microrganismos e seus genomas, possuindo grande importância na homeostase e aparecimento de distúrbios, assim como o TEA. A microbiota mantém a barreira da mucosa produzindo nutrientes (como vitaminas) e defendendo o organismo de patógenos. Logo, é necessária uma microbiota saudável para melhorar o desenvolvimento dos sistemas metabólicos e neurológicos (ROUSSIN L, et al., 2020).

A microbiota intestinal pode ainda afetar o neurodesenvolvimento por meio da via bidirecional entre 0 cérebro e o intestino. Esses microrganismos estimulam a diferenciação celular do trato gastrointestinal e, a partir disso, ocorre a liberação de neurotransmissores e hormônios, além da produção de metabólitos 
neuroativos que prejudicam o sistema nervoso central ou influencia o nervo vago. Com isso, o comportamento do indivíduo será afetado, representando uma ligação entre as áreas emocionais e cognitivas do cérebro com as funções intestinais periféricas, sugerindo sua influência na patogênese do autismo (PERETTI S, et al., 2018). Ressalta-se ainda que a prevalência de sintomas gastrointestinais é quatro vezes maior em crianças com TEA do que em crianças com desenvolvimento típico (DT).

De acordo com Hsiao EY (2014), os portadores de TEA possuem problemas gastrointestinais significativos e estudos revelam que esse distúrbio está fortemente associado à disbiose, ou seja, uma alteração na microbiota intestinal dessas crianças, contribuindo para deficiências cognitivas e comportamentais. Essa mudança da microbiota em comparação com as crianças em desenvolvimento típico (DT) é caracterizada por baixo nível de Bifidobacterium, firmicutes e alto nível de clostridium, bacteroidetes, desulfovibrio e lactobacillus.

Os clostrídios, por exemplo, produzem uma neurotoxina que inibe a liberação de neurotransmissores e contribui para a ansiedade, estresse e outros sintomas característicos de portadores de TEA. Assim, a disbiose é associada a dificuldades significativas de cognição, humor e dor, causando comportamentos irritativos nas crianças do espectro (HSIAO E, 2014). O TEA, que antes tinha pouca visibilidade, hoje é visto como uma disfunção do sistema nervoso central (SNC) com distúrbios no corpo em geral, incluindo o trato digestivo. Segundo Srikantha P e Mohajeri M (2019), há uma conexão importante entre a microbiota intestinal e o sistema nervoso central (SNC), e a modulação do eixo microbiota-intestino-cérebro pode ser uma forma eficaz e promissora para melhorar os sintomas da doença.

Assim, o presente artigo tem como objetivo evidenciar através de uma revisão narrativa sobre a íntima relação das adversidades gastrointestinais com a qualidade de vida de crianças portadoras do TEA.

\section{REVISÃO BIBLIOGRÁFICA}

\section{Aspectos do desenvolvimento cognitivo e social}

O TEA é uma complexa doença hereditária, que pode resultar de disfunções cerebrais decorrentes de interação de fatores ambientais, genéticos e epigenéticos (JOHNSON CP, et al., 2007). É caracterizado como uma síndrome ou um transtorno neurobiológico complexo que acomete o comportamento de seu portador, podendo ser diagnosticado a partir dos 12 meses e pode comprometer de diversas maneiras o desenvolvimento mental do paciente, afetando assim, negativamente a sua saúde e o seu bem-estar (RISTORI M, 2019). O Transtorno do Espectro do Autismo (TEA) é um transtorno do desenvolvimento neurológico, caracterizado por dificuldades de comunicação e interação social e pela presença de comportamentos e/ou interesses repetitivos e restritos.

Sendo assim, são caracterizados por distúrbios de fala e linguagem, padrões restritos de estresse, disfunções motoras e deficiências tanto intelectuais, quanto de aprendizagem. Contudo, o autismo tem uma alta variabilidade em relação à presença e a intensidade de seus sintomas, podendo indicar que tenham subtipos adicionais (RISTORI M, 2019). Para o diagnóstico da doença necessita-se de uma avaliação mais abrangente, integrada por uma abordagem passo a passo do paciente para que se tenha sua história detalhada e completa, bem como um exame bem detalhado e aplicação de critérios de diagnóstico para TEA do Manual Diagnóstico e Estatístico de Transtornos Mentais (MUKHERJEE SB, et al., 2017).

O diagnóstico é feito pelo uso do Modifield Checklist for Autism in Toddlers (M-CHAT). Esses pacientes também podem portar uma série de outras doenças associadas, como hiperatividade, epilepsia, distúrbios do sono e gastrointestinais, sendo o último o mais explorado nesse artigo (OLIVEIRA KG, et al., 2017).

Quanto a etiologia do TEA pode-se dividir os portadores em dois grupos. O primeiro grupo é denominado como forma primaria ou essencial, na qual não há uma causa específica para o transtorno. Já o segundo grupo é denominado como forma secundária ou sindrômica, no qual a causa do transtorno é identificada. Essa forma secundária, também, possui duas outras subdivisões, sendo uma caracterizada por fatores 
genéticos, gerada por anomalias cromossômicas e defeitos estruturais do genoma, a outra é caracterizada por fatores ambientais, como infecções, intoxicações fetais e fatores que entrem em contato com o genoma por meio de mecanismos epigenéticos (ALMEIDA SSA, et al., 2018).

Os distúrbios gastrointestinais em crianças com TEA pode gerar uma associação ao agravamento dos sintomas do transtorno. Fatores como ranger os dentes, caretas, compressões abdominais, soluços, mastigação excessiva entre outros. Crianças com TEA que apresentem dores estomacais ou desconforto abdominal pode ser um indicativo de excitação motora, que pode acabar tendo efeito em fatores comportamentais do paciente, como irritabilidade, distúrbios do sono e restrições alimentares (KLUKOWSKI M, et al. 2015).

\section{Sintomas gastrointestinais e qualidade de vida}

A relação do TEA com os distúrbios gastrointestinais é cada vez mais consensual na literatura moderna, sendo considerada uma síndrome clínica de sobreposição de sintomas, decorrente das diferentes condições fisiopatológicas genéticas e ambientais pertinentes à integração da microbiota intestinal com o cérebro, influenciando diretamente qualidade de vida do paciente autista (WASILEKSKA J e KLUKOWSKI M, 2015).

Uma metanálise publicada por McElhanon OB, et al. (2014), envolvendo 2215 crianças em 15 estudos, mostrou que portadores do TEA apresentam uma ocorrência de sintomas gastrointestinais quatro vezes maior do que aqueles que não são autistas. Dentre os sintomas mais descritos na análise comparativa entre indivíduos autistas em relação não portadores do TEA a partir desse estudo estão: a diarreia com razão de chances de 3,63 (IC 95\%, 1,82 - 7,23); constipação com razão de chances de 3,86 (IC 95\% 2,23 - 6,71); e dor abdominal com razão de chances de 2,45 (IC de 95\%, 1,19-5.07), todas em favor dos portadores do autismo. Outros sintomas gastrointestinais descritos, mas de menor prevalência, incluem refluxo gastresofágico, podendo ocorrer esofagite, vômitos, fezes sanguinolentas e flatulências (BUIE T, et al., 2010).

São levantadas diversas hipóteses sobre as possíveis etiologias dos sintomas como a menor tolerância à digestão de carboidratos, aumento da permeabilidade intestinal, presença de estado inflamatório sistêmico com ocorrência de gastrite e enterocolite, alergias alimentares, doença do refluxo gastroesofágico e dismotilidade intestinal (WASILEKSKA J e KLUKOWSKI M., 2015).

Contudo, em casos de constipação, cerca de 95\% das situações não estão relacionadas a patologias, sendo ocasionadas por causas funcionais oriundas do comportamento ou da disautonomia do sistema nervoso entérico (SNE) que compõe o sistema nervoso autônomo (SNA), também vista em outras afecções neuropsiquiátricas como foi descrito por McElhanon OB, et al. (2014). A principal hipótese é que a mesma seja causada por danos ao sistema límbico, manifestando-se em agitação ou até mesmo convulsão, como reações reflexas na tentativa de evitar sensações desagradáveis (WASILEKSKA J e KLUKOWSKI M, 2015).

Em 2016, foi descartado o papel dos peptídeos opioides na ocorrência dos sintomas digestivos nos portadores do TEA, uma vez que seus níveis nunca foram encontrados aumentados no SNC. Entretanto, o trabalho enfatizou a relação do estresse oxidativo promovido pelas bactérias da microbiota intestinal alterada, que aumenta a permeabilidade intestinal, sendo os pacientes autistas mais suscetíveis à absorção e consequente penetração pela barreira hematoencefálica de citocinas inflamatórias e toxinas, causadoras de mudanças comportamentais nesses indivíduos (LÁZARO PC, et al., 2016).

Por outro lado, estudos mais recentes descartaram que a ocorrência da inflamação intestinal promova inflamação sistêmica com altos níveis de citocinas pró-inflamatórias como o TNF- alfa; IL-1; e IL-6, exceto pelos altos níveis da resistina que foi implicada na ocorrência de diversos distúrbios inflamatórios do SNC em pacientes autistas, contraindicando assim terapia anti-inflamatória para pacientes autistas (PROSPERI M, et al., 2019).

Os sintomas gastrointestinais tornam-se um objeto desafiador no cuidado do paciente autista, uma vez que sua interpretação é dificultada pelas dificuldades comunicativas pertinentes ao transtorno, podendo ser precipitados ou agravados por situações de fuga à rotina do mesmo, como casos de constipação ocorridos devido às mudanças ou frustrações em seus hábitos fisiológicos (MCELHANON OB, et al., 2014). 
Além disso, outro aspecto desafiador torna-se a seletividade de sabores, cores e texturas de alimentos; e exclusividade nos subtipos pertencentes a sua dieta, dado que a restrição ao consumo de determinadas classes alimentícias, como frutas e vegetais, possa influir negativamente na motilidade intestinal. Sendo assim, uma vez conhecido o papel dos nutrientes na regulação da microbiota intestinal, o cardápio desses indivíduos deve ser ajustado e incrementado de acordo com as necessidades nutritivas e com as restrições de alimentos baseando nas manifestações de tolerâncias ou alergias desses pacientes (RISTORI MV, et al., 2019).

Podem ocorrer situações comportamentais decorrentes de algum desconforto em decorrência dos distúrbios gastrointestinais, entre elas: o ranger dos dentes, caretas faciais, mastigação excessiva, soluço, gritos, ecolalia, entre outros (BUIE T, et al., 2010). Por outro lado, porém, podem explicar mudanças súbitas de comportamento como agitação súbita, irritabilidade e privação do sono ou exacerbar condições de base, podendo indicar problema gastrointestinal subjacente, devendo então ser investigados (MCELHANON OB, et al., 2014).

Além disso, podem ocorrer os chamados comportamentos abdominais, com realização de pressão do abdome pelas mãos ou objetos; e os comportamentos motores com movimentos grosseiros e estereotipados, os quais são mais preocupantes devido ao risco de agressão e automutilação. Em relatório de consenso em 2010, foi apresentado ainda que crianças com dor abdominal são mais propensas a distúrbios psicológicos, ansiedade e sintomas comportamentais alterados (BUIE T, et al., 2010). Outros estudos demonstraram, contudo, não haver relação entre sintomas gastrointestinais e movimentos estereotipados e auto lesivos, sendo mais características alterações no sono e desenvolvimento cognitivo, como a linguagem, do que comportamentos agressivos (WASILEKSKA J e KLUKOWSKI M., 2015).

\section{Eixo microbiota-intestino-cérebro e alterações no TEA}

No TEA é observado dificuldade no aprendizado da linguagem e escassez de sociabilidade, sendo assim caracterizado como uma disfunção do neurodesenvolvimento. Esse distúrbio autista é considerado do eixo cérebro-intestino por $70 \%$ dos portadores de autismo possuírem sintomas abdominais. Com isso, inúmeras revisões descrevem que distúrbios como o autismo são moduladas, disfunções fisiológicas e comportamentais, pela microbiota pois na primeira infância essa microbiota encontra-se desregulada e assim leva a disfunções cerebrais (TIMOTHY G, et al., 2016).

A microbiota intestinal é importante para a saúde intestinal e de órgãos como o cérebro. Caso ela esteja alterada, como ocorre na disbiose, pode gerar impactos significativos no funcionamento do cérebro, por ter os metabólitos essenciais não produzidos de forma adequada pelos microrganismos intestinais. Além disso, o eixo cérebro-intestino-microbiota é onde ocorre a comunicação entre esses microrganismos com o cérebro e do cérebro com o sistema gastrointestinal sendo visto que a microbiota intestinal regula, sintetiza e libera alguns neurotransmissores centrais (TIMOTHY G, et al., 2016).

Em um estudo realizado por Strati $F$ (2017), constatou-se a relação direta entre os microorganismos intestinais e os neurônios entéricos na produção de produtos químicos como serotonina, dopamina, quinurenina e y-ácido aminobutírico e na regulação do eixo HPA. A disbiose de um grupo de microorganismos pode gerar uma inflamação sistêmica pelo aumento da ativação das respostas das células T-helper e T-helper 1 o que afeta as células imunológicas do SNC.

Além disso, nos transtornos do espectro autismo (ASDs) foi visto a ocorrência de uma ativação do sistema imunológico precocemente com inflamação crônica e falha na regulação de citocinas o que nos chama mais atenção a esses eventos gastrointestinais. Por fim, foi visto que a constipação é um sintoma do sistema gastrointestinal frequentemente relatado por indivíduos autistas e essa constipação agrupa diferentes tipos bacterianos. Essas comunidades de microorganismos intestinais alteradas não se relacionam ao modo de constipação dos portadores de autismo e sim dependem do próprio transtorno autista (STRATI F, et al., 2017).

\section{Dietas, evidências sobre nutrientes específicos e outras intervenções}

As terapias de primeira linha para o TEA são baseadas em fonoaudiologia, terapia ocupacional, comportamental aplicada e programas educacionais. Porém, alguns estudos indicam que a associação com 
intervenções nutricionais pode ser usada como tratamento adjuvante, com observação de bons resultados relacionados ao comportamento e anormalidades gastrointestinais (KARHU E, et al., 2019).

Crianças com TEA são comumente seletivas na alimentação. Geralmente elas preferem amido, lanches e alimentos processados e recusam frutas, vegetais e proteínas. Normalmente essas crianças têm níveis diminuídos de ácido pantotênico, folato, biotina, vitaminas, principalmente B12, D e E, e também redução do cálcio, magnésio, cobre e ferro. Além disso, é comum a ocorrência de intolerâncias alimentares, principalmente por laticínios, nozes e frutas, que podem produzir uma reação do sistema imunológico e provocar liberação de citocinas (químicos inflamatórios), os quais podem inflamar o cérebro (PERETTI S, et al., 2018).

Alguns estudos sugerem que intervenções nutricionais podem ser uma via de tratamento dos sintomas comportamentais e gastrointestinais em crianças com TEA (PERETTI S, et al., 2018). As principais dietas utilizadas são sem glúten e sem caseína (GFCF), cetogênica, com ferro, ácido graxo (suplementação de ômega 3), dieta específica de carboidrato, suplementos alimentares como vitaminas A, E, B12, além de magnésio e folato (KARHU E, et al., 2019).

Porém, ainda não existe embasamento científico para justificar certas restrições alimentares, podendo até causar danos nutricionais devido à maior seletividade alimentar apresentada por crianças com TEA. A dieta GFCF é justificada devido a efeitos dos peptídeos opióides, que são liberados na digestão dessas proteínas, os quais diminuem a captação de cisteína pelas células, consequentemente gera níveis reduzidos de glutationa pois eles são limitantes para síntese de GSH, além disso diminuem o índice de metilação. Dessa forma, essa dieta favoreceria a absorção de cisteína e consequentemente os níveis de glutationa, que quando está reduzida no trato gastrointestinal pode promover inflamação, contribuindo para o desconforto no TEA (LÁZARO P, et al., 2016).

Segundo Peretti S, et al. (2018), outro tipo é a dieta cetogênica que mostrou uma remodelação do microbioma intestinal e progresso nos sintomas neurológicos como melhoria nas habilidades cognitivas e sociais, funções de linguagem e uma resolução de comportamentos estereotipados. No entanto, mais estudos são necessários para entender melhor esse processo terapêutico.

Foi demonstrado ainda que a deficiência de vitaminas, magnésio e folato associado aos ácidos graxos em crianças com autismo é um achado comum e a suplementação desses nutrientes pode ajudar na redução do estresse oxidativo, nos sintomas gastrointestinais do TEA. Ademais, a deficiência simultânea deles, embora associada a baixa ingestão, também pode se relacionar com a disbiose intestinal reduzindo a sua absorção. (PERETTI S, et al., 2018).

Entretanto, para as crianças que possuem seletividade alimentar, é importante ter cuidado ao se implementar essas dietas com exclusão de itens alimentares, de forma a garantir uma ingestão nutricional adequada (KARHU E, et al., 2019).

Outras estratégias como o uso de prebióticos e probióticos, baseadas na ideia de que a dieta influencia as bactérias intestinais, têm demonstrado papel importante na digestão alimentar, com melhora do humor, ansiedade e estresse nesses pacientes (PERETTI S, et al., 2018).

Além disso, verificou-se também uma ligação entre a microbiota materna e distúrbios do neurodesenvolvimento, uma vez que a microbiota do feto começa com os tecidos do útero, ou seja, dieta materna ou o estresse durante a gravidez podem alterar negativamente o desenvolvimento da criança. Diante disso, existem recomendações sobre o uso de prebióticos para reduzir a chance de desenvolver autismo, porém novos estudos são necessários para confirmar essa possibilidade (PERETTI S, et al., 2018).

Outra intervenção, proposta por Kang DW, et al. (2019), é o transplante de microbiota fecal (TMF) que é um tratamento com vancomicina de duas semanas, associado a limpeza intestinal e altas doses de TMF por 1-2 dias e 7-8 semanas junto com supressor de ácido estomacal administrados em crianças com TEA, diversificando as bactérias presentes no intestino desses pacientes. Após 2 anos concluíram que essas crianças reduziram seus sintomas gastrointestinais e do autismo. 
De acordo com Peretti S, et al. (2018), a dieta materna, a qual pode ser deficiente ou excessiva, é grande influenciadora para o desenvolvimento subsequente do autismo, sendo os principais elementos o ácido fólico, ácidos graxos poliinsaturados, vitaminas, metanol e aspartame.

É sabido que durante a gestação as demandas metabólicas aumentam, assim, existe uma recomendação de se suplementar o ácido fólico neste período devido aos baixos níveis de folato encontrados durante a gravidez. Essas recomendações são baseadas em duas hipóteses, cuja primeira está relacionada a redução do risco de defeitos do tubo neural, atraso de linguagem e hiperatividade, já a segunda se baseia na melhora das competências sociais, atenção e funções executivas, sendo estes os déficits que caracterizam os indivíduos com autismo (PERETTI S, et al., 2018).

Outro ponto abordado está relacionado aos ácidos graxos poliinsaturados, que são fatores chaves para o crescimento e desenvolvimento do cérebro cuja deficiência pode estar ligada ao desenvolvimento do autismo. Pois eles afetam principalmente o córtex pré-frontal e os circuitos subcorticais (área límbica), onde essas áreas estão comprometidas em diversos transtornos como o TEA (PERETTI S, et al., 2018; KARHU E, et al., 2019).

Ademais, segundo Cheng B, et al. (2020), a vitamina A também desempenha papel crítico no desenvolvimento inicial do cérebro, proliferação e diferenciação neuronal e crescimentos neuritos e sinapses. Foi estudado ainda que a exposição a alimentos contendo metanol e aspartame e também com alto teor de gordura durante a gravidez pode estar associada a um risco maior de TEA e outros distúrbios do desenvolvimento neurológico (PERETTI S, et al., 2018).

\section{CONSIDERAÇÕES FINAIS}

Esta revisão forneceu evidências sugestivas de que a terapia nutricional em crianças com TEA é uma opção de tratamento eficiente para sintomas gastrointestinais e comportamentais, visto que essa condição causa uma redução significativa da qualidade de vida devido a prevalência dos sintomas gastroentéricos que associados ao TEA causam comportamento irritativos nas crianças do espectro. Verificou-se também a importância de uma abordagem diferenciada em relação ao alimento na dieta de pessoas com TEA, devido à alta seletividade alimentar das crianças dentro do espectro autista que acabam cursando com excesso ou carência de nutrientes, o que frequentemente leva ao ganho de peso excessivo e consequentemente ao desenvolvimento de obesidade. No entanto, ainda é necessário que sejam elaborados mais estudos para investigar quais seriam as melhores abordagens e benefícios dessa intervenção, para que possam servir de subsídio para os profissionais atuarem de forma efetiva no cuidado de pessoas com TEA.

\section{REFERÊNCIAS}

1. ALMEIDA SSA, et al. Transtorno do espectro autista. Revista do peiatra, 2018; 8 (spl. 1).

2. BUIE T, et al. Evaluation, Diagnosis, and Treatment of Gastrointestinal Disorders in Individuals with ASDs: A Consensus Report. Pediatrics, 2010; 125 (s.1): 1-18.

3. CHENG B, et al. Vitamin A deficiency increases the risk of gastrointestinal comorbidity and exacerbates core symptoms in children with autism spectrum disorder. Pediatric, 2020.

4. HSIAO E. Gastrointestinal issues in autism spectrum disorder. Harvard review of psychiatry, 2014; $22(2): 104-111$.

5. JAGGAR $M$, et al. You've got male: sex and the microbiota-gut-brain axis across the lifespan. Frontiers in neuroendocrinology, 2020; 56: 100815.

6. JOHNSON CP, et al. Identification and Evaluation of Children with Autism Spectrum Disorders. Pediatrics, 2007; 120 (5): 1183-1215.

7. KANG DW, et al. Long-term benefit of Microbiota Transfer Therapy on autism symptoms and gut microbiota. Scientific Reports, 2019.

8. KLUKOWSKI M, et al. Sleep and gastrointestinal disturbances is autism spectrum disorder in children. Developmental Perios Medicine, 2015; 19 (2): 157-161.

9. KARHU E, et al. Nutritional interventions for autism spectrum disorder. Nutrition Reviews, 2020; 78(7): 515-531.

10. LÁZARO P. Cristiane et al. Opioid peptides and gastrointestinal symptoms in autismo spectrum disorders. Revista Brasileira de Psiquiatria, 2016; 38: 243-246.

11. MCELHANON OB, et al. Gastrointestinal Symptoms in Autism Spectrum Disorder: A Meta-analysis. Pediatrics, 2014; 133(5): 872-883.

12. MUKHERJEE SB. Autism Spectrum Disorders — Diagnosis and Management. Indian J Pediatr., 2017; 84: 307-314. 
13. OLIVEIRA KG, SERTIÉ AL Transtornos do espectro autista: um guia atualizado para aconselhamento genético. Einstein, 2017; 15: 233-238.

14. OMS. Folha informatia - Transtorno do espectro autista. Brasília, 2017.

15. PERETTI S, et al. Diet: the keystone of autism spectrum disorder?. Nutritional neuroscience, 2019; 22(12): 825-839.

16. PROSPERI M, et al. Inflammatory Biomarkers are Correlated with Some Forms of Regressive Autism Spectrum Disorder. Brain Sci., 2019; 9(366): 1-14.

17. RISTORI M. Vitoria et al. Autism, Gastrointestinal Symptoms and Modulation of Gut Microbiota by Nutritional Interventions. Nutrients, 2019; 11(2812).

18. ROUSSIN L, et al. Role of the Gut Microbiota in the Pathophysiology of Autism Spectrum Disorder: Clinical and Preclinical Evidence. Microorganisms, 2020; 8(9): 1369.

19. SRIKANTHA P, MOHAJERI M. Hasan. The possible role of the microbiota-gut-brain-axis in autism spectrum dinternational journal of molecular sciences, 2019; 20(9): 2115.

20. STRATI F, et al. Novas evidências sobre a microbiota intestinal alterada em transtornos do espectro do autismo. Microbione, 2017; 5; 1-11.

21. TIMOTHY G, et al. O Microbiome-Gut-Brain Eixo na Cura e na Doença. Article in press, 2016; 16; 1-13.

22. WASILEWSKA J, KLUKOWSKI M. Gastrointestinal symptoms and autism spectrum disorder: links and risks-a possible new overlap syndrome. Pediatric health, medicine and therapeutics, 2015; 6: 153. 